\title{
Preferences of patients and health professionals for route and frequency of administration of biologic agents in the treatment of rheumatoid arthritis
}

\author{
This article was published in the following Dove Press journal: \\ Patient Preference and Adherence \\ 20 January 2014 \\ Number of times this article has been viewed
}

\author{
Tuan Khai Huynh ${ }^{1,2}$ \\ Ann $\varnothing_{\text {stergaard }}{ }^{3}$ \\ Charlotte Egsmose ${ }^{3}$ \\ Ole Rintek Madsen ${ }^{3}$ \\ 'Department of Rheumatology, \\ Copenhagen University Hospital \\ Frederiksberg, Frederiksberg, \\ Denmark; ${ }^{2}$ Department of \\ Rheumatology, Copenhagen University \\ Hospital Hillerød, Hillerød, Denmark; \\ ${ }^{3}$ Department of Rheumatology/C, \\ Copenhagen University Hospital \\ Gentofte, Hellerup, Denmark
}

Objectives: To examine the preferences of rheumatoid arthritis (RA) patients and health professionals (HPs) for the route and frequency of administration of biologic drugs.

Methods: One hundred and seven RA patients treated with biological agents for intravenous or subcutaneous use, 35 biologic-naïve RA patients treated with a disease-modifying antirheumatic drug and 30 rheumatology HPs (physicians and nurses) were recruited from two outpatient clinics in Copenhagen, Denmark. All subjects filled out a questionnaire interrogating their choice of preferred route and frequency of administration of a biologic corresponding to current available options, given that effects, adverse effects, and financial costs were identical for the different choices. The subjects were also asked to justify their preferences. The chi-square goodnessof-fit test was used to examine the distributions over different preferences. Proportions were compared using Fisher's exact test.

Results: Forty-one patients were currently treated with subcutaneous self-injections at home (SCH) and 66 intravenously at the clinic (IVC). IVC was preferred by $85 \%$ of patients currently treated with IVC $(P<0.0001)$. SCH was preferred by $71 \%$ of patients currently treated with SCH $(P<0.001)$, by $77 \%$ of the biologic-naïve patients $(P<0.01)$, and by $87 \%$ of HPs $(P<0.0001)$. The proportion of patients favoring $\mathrm{SCH}$ was significantly higher for patients currently receiving SCH and for biologic-naïve RA patients than for those currently on IVC $(P<0.0001)$. SCH once a month and IVC every 8 weeks were the most appealing treatment frequencies $(P<0.01)$. The most frequent reason among patients for choosing IVC or SCH was a wish for safety, and a wish to minimize the time of transportation and treatment, respectively.

Conclusion: The majority of RA patients treated with biologics preferred their current route of administration. Most patients, those inexperienced with biologics, and HPs favored SCH. Low treatment frequencies were generally preferred.

Keyword: biologic treatment, infusion, rheumatology, subcutaneous injection

\section{Introduction}

Rheumatoid arthritis (RA) affects approximately $1 \%$ of the population and is associated with increased morbidity and mortality. ${ }^{1,2}$ The main treatment goals include control over pain and inflammation, delaying the progression of bony erosions, and improving functional capacity. ${ }^{3}$ Treatment options have expanded significantly since the introduction of the biologic therapies 15 years ago, and several biologic agents are now available for subcutaneous or intravenous use. The frequency of administration depends on the specific biologic agent, but several options are available. ${ }^{4}$ The American College of Rheumatology and the European League Against Rheumatism have published
Correspondence: Ole Rintek Madsen Department of Rheumatology/C, Copenhagen University Hospital Gentofte, Copenhagen, Denmark Tel +4539778382

Fax +453977 7619

Email rintek@dadlnet.dk 
recommendations for the initiation of disease-modifying antirheumatic drugs (DMARDs) and biological agents for the treatment of RA. These recommendations include algorithms based on the efficacy of current treatment, the presence of poor prognostic factors (eg, positive anti-citrullinated peptide antibody and radiologic joint damage), and the level of disease activity. ${ }^{5,6}$ However, as the efficacy and toxicity of the biologic agents seems to be comparable in RA, ${ }^{7,8}$ as the route of administration may affect the adherence to the therapeutic regimen, ${ }^{9}$ and as patient and physician drug preferences may differ, ${ }^{10-13}$ it could be argued that priority should be given to the patient's choice of treatment. This would also be in agreement with guidelines recommending the involvement of RA patients in decision making ${ }^{14}$ and outcome assessment. ${ }^{15}$ Furthermore, the increasing pressure from society to involve patients in decisions about their own care emphasizes the need for studies on patient preference and adherence. In this context, the potential impact of attitudes among rheumatology health professionals (HPs) to available treatment options on patient preferences within the field of RA also needs further investigation. The data on the preferences of RA patients for the route and frequency of administration of biologic agents are very limited but do indicate that subcutaneous treatment may be preferred. ${ }^{16-18}$ The preferences of rheumatology HPs have not been examined.

The objectives of the present study were 1) to examine the preferences of biologic-naïve and non-naïve RA patients for the route and frequency of administration of biologic agents, and 2) to evaluate the same preferences of rheumatology HPs when considering their own need for treatment.

\section{Methods}

\section{Participants}

One hundred and seven consecutive RA patients treated with infliximab, abatacept, or tocilizumab (intravenously administered), or by etanercept or adalimumab (subcutaneously administered), and 35 consecutive biologic-naïve RA patients treated with synthetic DMARDs (administered orally) (referred to as the "DMARD patients") for at least 6 months were recruited from two university outpatient clinics in Copenhagen serving urban rheumatology patients. Patients treated intravenously once a year (with rituximab) or subcutaneously once a month (with golimumab) were not included as the number of patients was considered too low. Thirty rheumatology HPs (rheumatologists and nurses) from the same outpatient clinics also volunteered to participate. Improved consent was obtained from all participants. According to the scientific ethics committee for Copenhagen, ethics approval was not required for this type of study. The study was conducted in accordance with the Helsinki Declaration of 1975, as revised in 1983.

\section{Questionnaire}

A paper and pencil questionnaire was designed specifically for use in the present study as no usable questionnaire was found in the literature. The questionnaire was self-explanatory and included easily understood user instructions. This allowed self-administration with only a minimum need for verbal instructions. All subjects filled out the questionnaire interrogating their choice of the preferred route and frequency of administration of a nameless biological agent corresponding to the currently available options (except for the twice a week frequency), given the premise that the effects, adverse effects and financial costs were identical for the different choices. The HPs were asked to answer the questionnaire as though they themselves were to be treated as patients with RA. The questionnaire was to be completed within 15 minutes, at the outpatient clinic, without any interference from HPs or colleagues.

The questionnaire consisted of three parts. In the first part, the participants were asked to choose one of the following options for the route and frequency of administration: intravenous infusion at the outpatient clinic (IVC) every 8 weeks; IVC every 4 weeks; two IVC, 2 weeks apart, once a year; subcutaneous self-injection at home ( $\mathrm{SCH}$ ) once a week; $\mathrm{SCH}$ every other week; $\mathrm{SCH}$ once a month; and $\mathrm{SCH}$ with the help of a home nurse once a week, every other week, or once a month. In the second part of the questionnaire, the participants were asked to justify their preference by choosing one or more of the following statements: "more easy to manage"; "more safe to get an infusion at the hospital"; "more comfortable with self-injections at home"; "appreciate the social contact at the hospital"; "dislike hospitals"; and "wish to minimize time of transportation and treatment" and if "yes", "because of functional impairment." In the third section, participants were asked to write down the transportation time to the hospital.

\section{Statistics}

The data are presented as the numbers and proportions and by the mean \pm standard deviation (SD) or the median (range). The chi-square goodness-of-fit test was used to test whether the observations were uniformly distributed over the different preferences. The proportions were compared using Fisher's exact test. The continuous variables were compared using Student's $t$-test for unpaired data. The calculations were performed using the software package SPSS/PC+ Statistics V. 4.01 (SPSS, Inc, 
Chicago, IL, USA). Two-tailed $P$-values less than 0.05 were considered statistically significant.

\section{Results}

The mean age \pm SD was $57 \pm 14$ years for all patients, was $56 \pm 14$ years for the patients treated with a biological agent, and was $53 \pm 14$ years for the DMARD patients (difference not significant $[\mathrm{NS}])$. The mean age of the HPs was $49 \pm 9$ years $(P<0.05$, when compared with each of the two patient groups). Seventy-seven percent of the patients and $73 \%$ of the HPs were women (NS). The mean transportation time to hospital was $35 \pm 33$ (median 30, range 0-300) minutes for all participants. Forty-one patients were currently treated with adalimumab or etanercept $(\mathrm{SCH})$ and 66 with infliximab, tocilizumab, or abatacept (IVC). Eighty two percent of patients currently treated with $\mathrm{SCH}$ had previously been on IVC treatment. Fifteen percent of those treated with IVC had previously been on $\mathrm{SCH}$.

Fifty percent $(54 / 107)$ of the patients treated with a biologic agent reported preferring SCH treatment, and 50\% preferred IVC (53/107) (NS). Of those patients already treated with IVC, $85 \%$ preferred IVC treatment $(P<0.0001)$. SCH was preferred by $71 \%$ of patients currently treated with $\mathrm{SCH}$
$(P<0.001)$, by $77 \%$ of the DMARD patients $(P<0.01)$, and by $87 \%$ of HPs $(P<0.0001)$. The proportion favoring IVC was significantly higher for patients currently receiving IVC than for patients on SCH or DMARDs and HPs $(P<0.0001)$. The priorities for the route of administration for patients currently treated with SCH, IVC, or only DMARDs and for HPs are shown in detail in Figure 1, together with the results of the comparisons of the proportions. The HPs had a shorter transportation time than did the patients $(21 \pm 10$ versus $37 \pm 36$ minutes $)(P<0.05)$. The transportation time was similar for patients preferring IVC and $\mathrm{SCH},(38 \pm 43$ versus $37 \pm 28$ minutes, respectively) (NS). Age was also similar for these two patient groups ( $56 \pm 14$ versus $55 \pm 14$ years) (NS).

Among the patients who reported a preference for IVC treatment $(n=62), 15 \%$ preferred IVC with the shortest possible frequency, once a month; $51 \%$ favored infusion every 8 weeks; and 34\% favored two infusions, once a year $(P<0.01$ when comparing the proportions). Of the patients who preferred SCH treatment $(n=80), 53 \%$ chose the longest possible treatment interval, once a month; $32 \%$ chose injection once every 2 weeks; and $15 \%$ chose injection once a week $(P<0.001$ when comparing the proportions). Eighteen percent of the patients favoring $\mathrm{SCH}$ reported preferring a home nurse to

Prefer SCH $\square$ Prefer IVC

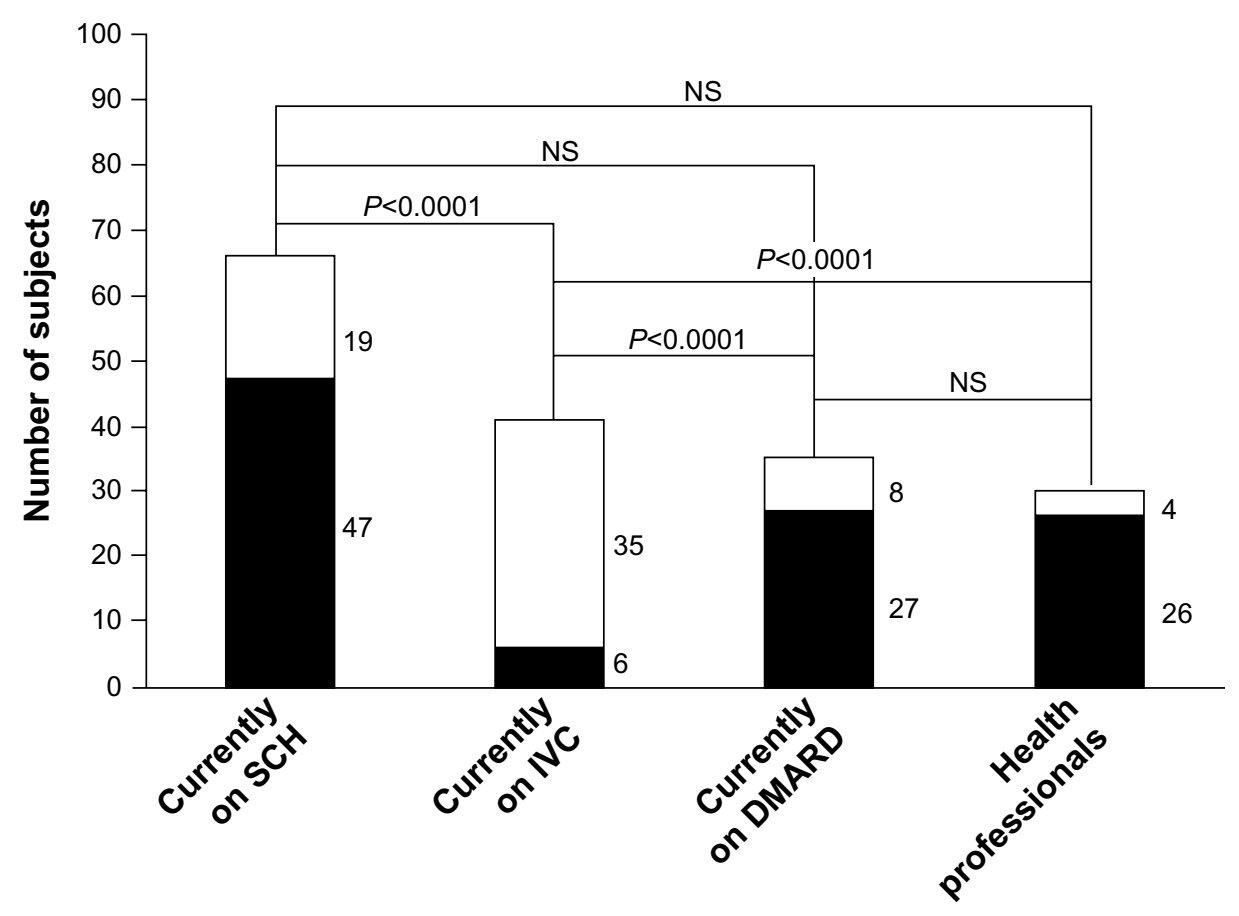

Figure I Number of patients and HPs preferring SCH or IVC.

Note: Patients are categorized according to the current mode of treatment administration.

Abbreviations: DMARD, disease modifying antirheumatic drug; HP, health professional; IVC, intravenous infusion at the outpatient clinic; NS, not significant; SCH, subcutaneous injection at home. 
give the injection if possible; in this subgroup, $93 \%$ preferred an injection, with a frequency of once a month.

Analyzing the subgroup of biologic-naïve (DMARD) patients preferring $\mathrm{SCH}(\mathrm{n}=27), 67 \%(\mathrm{n}=18)$ were found to favor injections given with a frequency of once a month and $33 \%(\mathrm{n}=9)$ with a frequency of once a week or every second week $(P=0.08)$; of these patients, $37 \%$ preferred a home nurse to give the injection, if possible. Eight of the DMARD patients reported a preference for IVC; none of these favored infusion once a month, $75 \%$ favored infusion only twice once a year, and 25\% favored infusion once every 8 weeks (NS).

Twenty-six of the 30 HPS preferred SCH treatment $(P<0.0001)$. Seventy-seven percent of these preferred injection only once a month $(P<0.01$; when compared to injection once weekly and every 2 weeks). All four HPs choosing IVC preferred infusion twice once a year.

The most frequent reason among patients as whole for choosing IVC treatment was "more safe" (65\%), followed by "easy to manage" (50\%). The two most frequent reasons for choosing SCH treatment were "minimize time of transportation and treatment" (63\%) and "more easy to manage" (43\%). The same pattern of reasons for choosing SCH or IVC was found for patients currently on biologics and for patients currently on DMARDs. The reasons for patients' preference for the route of administration are given in more detail for the total number of patients on biologics and DMARDs, respectively, in Table 1. The most frequent reason for choosing SCH among HPs was "comfortable with self-injections at home" $(85 \%)$ and "time for transportation and treatment" (65\%).

Table I Statements chosen by RA patients regarding preferences for route of administration of biologic agents (part two of the questionnaire)

\begin{tabular}{|c|c|c|c|}
\hline & \multicolumn{2}{|c|}{ Preference } & \multirow[t]{3}{*}{$P$} \\
\hline & $\mathrm{SCH}$ & IVC & \\
\hline & $n=80$ & $n=62$ & \\
\hline $\begin{array}{l}\text { More easy to manage preferred } \\
\text { administration }\end{array}$ & 43 & 50 & NS \\
\hline $\begin{array}{l}\text { More safe to get an infusion } \\
\text { at the hospital }\end{array}$ & 8 & 65 & $<0.0001$ \\
\hline $\begin{array}{l}\text { More comfortable with } \\
\text { self-injections at home }\end{array}$ & 38 & 5 & $<0.000$ I \\
\hline $\begin{array}{l}\text { Appreciate the social contact } \\
\text { at the hospital }\end{array}$ & 6 & 24 & $<0.005$ \\
\hline Dislike hospitals & 4 & 0 & NS \\
\hline $\begin{array}{l}\text { Wish to minimize time of } \\
\text { transportation and treatment }\end{array}$ & 63 & 31 & $<0.0001$ \\
\hline $\begin{array}{l}\text { If "yes" because of functional } \\
\text { impairment }\end{array}$ & 11 & 7 & NS \\
\hline
\end{tabular}

Note: Results are given as number of patients in percent.

Abbreviations: IVC, intravenous infusion at the outpatient clinic; NS, not significant; $\mathrm{RA}$, rheumatoid arthritis; $\mathrm{SCH}$, subcutaneous injection at home.

\section{Discussion}

The treatment options for patients with RA continue to expand, creating opportunities for improved outcomes, such as decreased pain, less disability, and decreased mortality. However, with the introduction of biologics, patients as well as physicians are faced with increasingly complex decisions about how and when a medication should be initiated and continued. At the same time, there is an urgent need to provide patients with individualized treatment strategies and to enable patient participation in shared medical decision making. ${ }^{19,20}$

Understanding patient needs provides the physician with the basis for the right therapeutic choice. ${ }^{21-23}$ Thus, assessing patient preferences for the route and frequency of medication administration in RA is a necessary step toward improving outcomes, by ensuring satisfaction and adherence. While the efficacy of the biologics has not been strictly evaluated in head to head trials, their route and frequency of administration differs and may play a role in decision-making for patients with RA. ${ }^{24}$ Financial costs and regional reimbursement regulations may also influence patients' and HPs' choice of treatment, but the present study was not aimed to study the importance of these aspects

Our study is unique as it examined the preferences of patients who were both inexperienced and experienced with biologic treatment as well as preferences among rheumatology HPs. As a whole, the patients preferred SCH. This was particularly the case for the DMARD patients, who were naïve to biological treatment, and for those currently treated with $\mathrm{SCH}$. Almost three-quarters of the patients currently treated with IVC preferred IVC. The fact that the majority of patients treated with SCH had prior experience with IVC demonstrates that SCH may turn out to be acceptable also for a large proportion of those patients currently treated with and preferring IVC. As regards to these patients, all had stopped IVC treatment due to lack of efficacy or side effects and had switched to an $\mathrm{SCH}$ treatment according to the current guideline of the department, without any specific focus on patient preferences for the route and frequency of administration. It is also noteworthy that only a minority of the patients favoring IVC preferred the shortest possible treatment interval, one month. On the other hand, one-third of patients favoring IVC were prepared to receive the infusions with the longest possible treatment interval, once a year, despite the fact than none of the patients were experienced with this regimen. Likewise, all of the patients currently treated with and favoring $\mathrm{SCH}$ were injected twice a week, every week (etanercept) or every other week (adalimumab), but the majority of these patients reported a preference for injections only once a month. 
These preferences for the type and frequency of administration demonstrate that patients already treated with a biologic may be rather conservative regarding the route of administration but may find a lower frequency of treatment more attractive. The longest possible interval between injections was also the most appealing choice for most of the DMARD patients, who were naïve to biological treatment.

The preferences for IVC and $\mathrm{SCH}$ showed that similar motives may lead to different choices. Approximately $50 \%$ of those preferring IVC and $40 \%$ of those preferring $\mathrm{SCH}$ felt that their choice of administration was the easiest to manage. However, different patient preferences were also characterized by different motives. A wish to minimize the time associated with treatment was far more often the reason for choosing $\mathrm{SCH}$ and the safety issue more often the reason for preferring IVC. The urban patients participating in the study were characterized by having a rather short travel time to the hospital, only 35 minutes on the average, with a narrow range except for some outliers. There was no difference in preference between the patients with the longest and shortest travel times, but it is reasonable to assume that longer travel times in other patient populations may possibly motivate more patients to choose $\mathrm{SCH}$ or alternatively, two infusions of IVC, once a year.

The results of the present study are in keeping with previous limited data. Using a questionnaire, Scarpato et $\mathrm{al}^{16}$ investigated the determinants of preference for the route of administration of anti-tumor necrosis factor (TNF) agents, in 802 anti-TNF-naïve patients with RA who were distributed nationwide in Italy. In that study, 98\% were treated with traditional synthetic DMARDs. The researchers found that the intravenous and subcutaneous routes of administration were both preferred by $50 \%$. The reasons given for the choice of intravenous administration were the safety of treatment at the hospital and the reassuring effect of the physician presence. The subcutaneous administration was chosen for the convenience of treatment and in particular of home treatment. In a study from the UK, ${ }^{17}$ subcutaneous or intramuscular administration was preferred over intravenous treatment by $82 \%$ of patients treated with synthetic DMARDS and by $65 \%$ of those treated with anti-TNF agents. The majority of subjects also preferred administration of the therapy at home. The preferences of the patients on anti-TNF therapy corresponded with the route and frequency of administration of the drug they were currently taking, suggesting they were satisfied with their treatment. In another study from UK, ${ }^{18}$ a questionnaire was sent to 200 subjects who were on combination DMARD therapy. Of the $56 \%$ who responded, $48 \%$ preferred self-injection versus $30 \%$ who did not, and $22 \%$ were unsure. A total of $41 \%$ preferred the rheumatologist to decide, reflecting that the opinion of the rheumatologist is of importance for many patients.

In any assessment of patient preference in the daily clinic, it is essential that patients have been fully informed of the risks and benefits, and of the route and frequency of administration of a particular treatment. Another important factor for effective, informed decision-making is trust in the physician since trust has been shown to have a greater effect on the patient's confidence in a DMARD decision than his or her DMARD-specific knowledge, disease-related factors, or demographics. ${ }^{25}$ However, clinicians are not always in a position to advice patients about treatment options based on what they would accept for themselves. ${ }^{10}$ At the same time physician preference has been shown to be an important determinant of patients' receipt of biological therapy. ${ }^{26}$ In the present study, the attitudes of rheumatologists and rheumatology nurses to route of administration and frequency of biologic treatment were examined for the first time. Furthermore, the results of the study may be particularly interesting in that it investigated these HPs' decisions regarding their own health care rather than what they would choose for their patients. A very positive attitude to the use of SCH with the longest possible interval between injections was demonstrated, although transportation time to the hospital was short. The HPs were slightly younger than the patients ( 49 versus 57 years). It has been reported that younger patients may prefer subcutaneous over intravenous therapy, ${ }^{18}$ but it is difficult to believe that age added significantly to the preference for $\mathrm{SCH}$ among HPs, especially as the patients preferring SCH and IVC had the same age. More likely, the HPs preferences were a reflection of their professional insight and confidence in their ability to self-manage treatment. The preferences of the HPs corresponded, by and large, to the preferences of the patients treated with DMARDs, who were inexperienced with biologics, and to the preferences of the patients currently treated with $\mathrm{SCH}$. On the other hand, a large proportion of the patients currently treated with IVC were satisfied and comfortable with this mode of administration. Thus, clinicians should be aware that some patients with RA may have other needs for care and may have other preferences than they would have themselves. However, it should also be noted that when biologic treatment has been initiated and proves to be effective, the treatment will not be changed in order to meet the patients' preference for another route or frequency of administration as switching may result in a poorer response. In the ideal world, however, the preferences of patients who are naïve to biological treatment and of patients with treatment failure should be taken into consideration before choosing 
a specific biologic agent and thereby, route and frequency of administration. Unfortunately for the patients, economic aspects may be the final determinant of treatment choice in many parts of the world.

The study has some limitations. The study population was relatively small, and patients were selected consecutively and not randomly. A larger sample would have allowed for a more detailed analysis of the subgroups, but it is difficult to believe that the sample was not representative of the patients and HPs in the capital region of Denmark. The patients' preferences of administration route and frequency may have been influenced by bad or good experience with current or previous treatments. However, conversely, it could be argued that experience with both IVC and SCH treatments resulted in more qualified preferences. In general, concerns regarding efficacy, side effects and financial burden may and should influence the choice of treatment. In Denmark, biological treatment is fully reimbursed by the state and the patients have no financial obligations. In any event, it was strongly pointed out to the participants that the effects, adverse effects, and financial costs should be considered identical for the different choices. Whatever the reason for preference, it would still be worthwhile to note that patients in general, were satisfied with the current administration. In Denmark, the administration of biological treatment by a home nurse is freely offered to patients who have psychological or physical self-injection difficulties. This is not a realistic option in many other countries. The study design (questionnaire survey) did not allow for the systematic assessment of timerelated disease activity, and reliable data on disease duration were not available in all cases. Hypothetically, the disease activity itself and disease duration may influence preferences for route and frequency of treatment in RA, although data on this issue are not available in the literature. Other parameters related to the patients, the disease, or the treatment, such as age, gender, and duration of current treatment, may also play a role in preference but were either not collected or were too complex to analyze in a meaningful way.

In conclusion, the majority of urban RA patients treated with biologics preferred their current route of administration but reported a preference for a lower treatment frequency. The majority of patients not currently treated with a biologic and the HPs in rheumatology favored SCH over IVC with a low treatment frequency. Safety issues were important to patients who preferred IVC.

\section{Acknowledgment}

We would like to acknowledge the patients, and the physicians and nurses who participated in this study.

\section{Disclosure}

Ole Rintek Madsen has received research grants and/or consultancy and speaker fees from Abbott, Bristol-Myers Squibb, Merck Sharp \& Dohme, Pfizer, Roche, and Union Chimique Belge. The authors report no other conflicts of interest.

\section{References}

1. Alamanos Y, Voulgari PV, Drosos AA. Incidence and prevalence of rheumatoid arthritis, based on the 1987 American College of Rheumatology criteria: a systematic review. Semin Arthritis Rheum. 2006;36(3):182-188.

2. Gabriel SE, Michaud K. Epidemiological studies in incidence, prevalence, mortality, and comorbidity of the rheumatic diseases. Arthritis Res Ther. 2009;11(3):229.

3. Keystone EC, Smolen J, van Riel P. Developing an effective treatment algorithm for rheumatoid arthritis. Rheumatology (Oxford). 2012;51 Suppl 5:v48-v54.

4. Smolen JS, Landewé R, Breedveld FC, et al. EULAR recommendations for the management of rheumatoid arthritis with synthetic and biological disease-modifying antirheumatic drugs. Ann Rheum Dis. 2010;69(6): 964-975.

5. Choy EH, Kavanaugh AF, Jones SA. The problem of choice: current biologic agents and future prospects in RA. Nat Rev Rheumatol. 2013;9(3):154-163.

6. Singh JA, Furst DE, Bharat A, et al. 2012 update of the 2008 American College of Rheumatology recommendations for the use of disease-modifying antirheumatic drugs and biologic agents in the treatment of rheumatoid arthritis. Arthritis Care Res (Hoboken). 2012;64(5):625-639.

7. Pierreisnard A, Issa N, Barnetche T, Richez C, Schaeverbeke T. Metaanalysis of clinical and radiological efficacy of biologics in rheumatoid arthritis patients naive or inadequately responsive to methotrexate. Joint Bone Spine. 2013;80(4):386-392.

8. Aaltonen KJ, Virkki LM, Malmivaara A, Konttinen YT, Nordström DC, Blom M. Systematic review and meta-analysis of the efficacy and safety of existing TNF blocking agents in treatment of rheumatoid arthritis. PLoS One. 2012;7(1):e30275.

9. Hetland ML, Christensen IJ, Tarp U, et al; All Departments of Rheumatology in Denmark. Direct comparison of treatment responses, remission rates, and drug adherence in patients with rheumatoid arthritis treated with adalimumab, etanercept, or infliximab: results from eight years of surveillance of clinical practice in the nationwide Danish DANBIO registry. Arthritis Rheum. 2010;62(1):22-32.

10. Montgomery AA, Fahey T. How do patients' treatment preferences compare with those of clinicians? Qual Health Care. 2001;10 Suppl 1: i39-i43.

11. Yazici Y, McMorris BJ, Darkow T, Rosenblatt LC. Patient and physician perception of the infusion process of the biologic agents abatacept, infliximab, and rituximab for the treatment of rheumatoid arthritis. Clin Exp Rheumatol. 2009;27(6):907-913.

12. Pullar T, Wright V, Feely M. What do patients and rheumatologists regard as an 'acceptable' risk in the treatment of rheumatic disease? Br J Rheumatol. 1990;29(3):215-218.

13. van Hulst LT, Kievit W, van Bommel R, van Riel PL, Fraenkel L. Rheumatoid arthritis patients and rheumatologists approach the decision to escalate care differently: results of a maximum difference scaling experiment. Arthritis Care Res (Hoboken). 2011;63(10):1407-1414.

14. Kennedy T, McCabe C, Struthers G, et al; British Society for Rheumatology Standards, Guidelines and Audit Working Group (SGAWG). BSR guidelines on standards of care for persons with rheumatoid arthritis. Rheumatology (Oxford). 2005;44(4):553-556.

15. Kirwan JR, Hewlett SE, Heiberg T, et al. Incorporating the patient perspective into outcome assessment in rheumatoid arthritis - progress at OMERACT 7. J Rheumatol. 2005;32(11):2250-2256. 
16. Scarpato S, Antivalle M, Favalli EG, et al; RIVIERA co-authors. Patient preferences in the choice of anti-TNF therapies in rheumatoid arthritis. Results from a questionnaire survey (RIVIERA study). Rheumatology (Oxford). 2010;49(2):289-294.

17. Williams EL, Edwards CJ. Patient preferences in choosing anti-TNF therapies-R1. Rheumatology (Oxford). 2006;45(12):1575-1576.

18. Chilton F, Collett RA. Treatment choices, preferences and decisionmaking by patients with rheumatoid arthritis. Musculoskeletal Care. 2008;6(1):1-14.

19. Fraenkel L, Fried TR. Individualized medical decision making: necessary, achievable, but not yet attainable. Arch Intern Med. 2010;170(6):566-569.

20. Fraenkel L, McGraw S. What are the essential elements to enable patient participation in medical decision making? J Gen Intern Med. 2007;22(5):614-619.

21. Cunha-Miranda L, Costa L, Ribeiro JS. NEAR study: Needs and Expectations in Rheumatoid ARthritis - do we know our patients needs? Acta Reumatol Port. 2010;35(3):314-323
22. Ho M, Lavery B, Pullar T. The risk of treatment. A study of rheumatoid arthritis patients' attitudes. Br J Rheumatol. 1998;37(4):459-460.

23. Marshall NJ, Wilson G, Lapworth K, Kay LJ. Patients' perceptions of treatment with anti-TNF therapy for rheumatoid arthritis: a qualitative study. Rheumatology (Oxford). 2004;43(8):1034-1038.

24. Goekoop-Ruiterman YP, de Vries-Bouwstra JK, Allaart CF, et al. Patient preferences for treatment: report from a randomised comparison of treatment strategies in early rheumatoid arthritis (BeSt trial). Ann Rheum Dis. 2007;66(9):1227-1232.

25. Martin RW, Head AJ, René J, et al. Patient decision-making related to antirheumatic drugs in rheumatoid arthritis: the importance of patient trust of physician. J Rheumatol. 2008;35(4):618-624.

26. Curtis JR, Chen L, Harrold LR, Narongroeknawin P, Reed G, Solomon DH. Physician preference motivates the use of anti-tumor necrosis factor therapy independent of clinical disease activity. Arthritis Care Res (Hoboken). 2010;62(1):101-107.
Patient Preference and Adherence

\section{Publish your work in this journal}

Patient Preference and Adherence is an international, peer-reviewed, open access journal focusing on the growing importance of patient preference and adherence throughout the therapeutic continuum. Patient satisfaction, acceptability, quality of life, compliance, persistence and their role in developing new therapeutic modalities and compounds to

\section{Dovepress}

optimize clinical outcomes for existing disease states are major areas of interest. This journal has been accepted for indexing on PubMed Central. The manuscript management system is completely online and includes a very quick and fair peer-review system. Visit http://www.dovepress.com/ testimonials.php to read real quotes from published authors.

Submit your manuscript here: http://www.dovepress.com/patient-preference-and-adherence-journal 